\title{
Correction to author list
}

Cite as: Can Urol Assoc J 2017;11 (7):E323. hitp://dx.doi.org/10.5489/cuaj.4775

Published online July 11, 2017.

In the article, "CUA guideline on adult overactive bladder," ${ }^{1}$ and the associated "Appendix: Executive summary of CUA guideline on adult overactive bladder," ${ }^{2}$ there was an error in the author listing. Correct authors are as follows:

Jacques Corcos, MD, FRCSC'; Mikolaj Przydacz, MD'; Lysanne Campeau, MD, PhD, FRCSC'; Jonathan Witten, MD'; Duane Hickling, MD, MSCl, FRCSC'; Christiane Honeine, RN, BN'; Sidney B. Radomski, MD, FRCSC ${ }^{4}$; Lynn Stothers, MD, MHSC, FRCSC ${ }^{5}$; Adrian Wagg, MD, FRCP (Lond), FRCP (Edin), FHEA ${ }^{6}$

'Department of Urology, Jewish General Hospital, McGill University, Montreal, QC; ${ }^{2}$ Northern Alberta Urology Centre, Edmonton, AB; ${ }^{3}$ Division of Urology, Department of Surgery, The Ottawa Hospital, Ottawa, ON; " Division of Urology, Toronto Western Hospital, University of Toronto, University Health Network, Toronto, ON; ${ }^{5}$ Department of Urological Sciences, University of British Columbia, Vancouver, $B C ;{ }^{6}$ Department of Medicine, University of Alberta, Edmonton, AB; Canada

\section{References}

1. Corcos J, Przydacz M, Campeau L, et al. CUA guideline on adult overactive bladder. Can Urol Assoc J 2017;11 (5):E142-73. http://dx.doi.org/10.5489/cuai.4586

2. Corcos J, Przydacz M, Campeau L, et al. Appendix: Executive summary of CUA guideline on adult overactive bladder. Can Urol Assoc J 2017;11 (5):E248-9. 